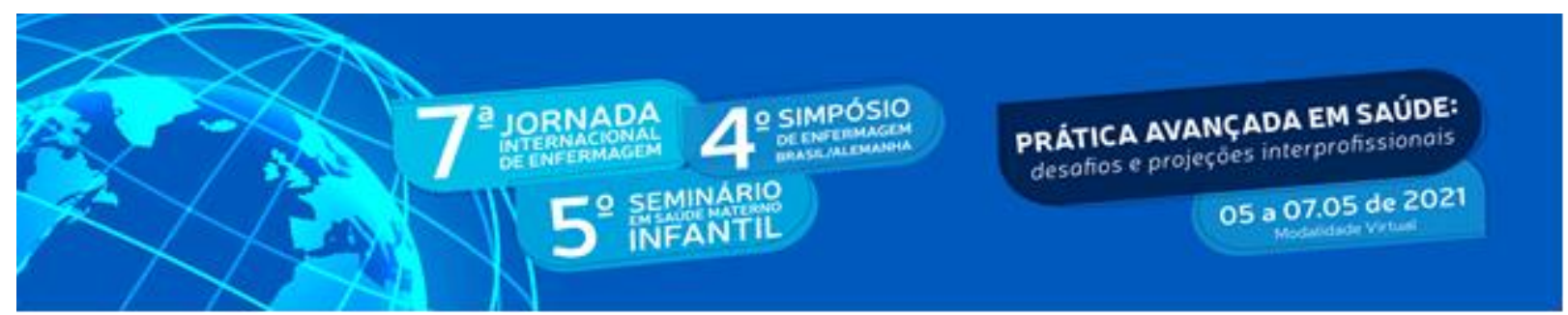

DOI: http://doi.org/10.48195/jie2021-113

\title{
TECNOLOGIA EM SAÚDE: PRODUÇÃO DE VÍDEO EDUCATIVO DOS MARCOS DO DESENVOLVIMENTO PARA PROFISSIONAIS DE ENFERMAGEM
}

\author{
Aline Mayra Mesquita Bello ${ }^{1}$; Jessica Maysa Oliveira Batista ${ }^{2}$; Layla Christine \\ Nascimento Alves ${ }^{3}$; Rosana de Jesus Santos Martins Coutinho ${ }^{4}$; Taynara de Jesus Costa \\ Conceição 5
}

\section{RESUMO}

Objetivo: Relatar a experiência da produção de vídeo educativo descrevendo os marcos do desenvolvimento infantil de 1 a 6 meses, a fim de promover formação aos profissionais de enfermagem da atenção primária à saúde, visando a efetiva avaliação dos marcos do desenvolvimento infantil. Metodologia: Estudo do tipo relato de experiência desenvolvido na disciplina de Saúde da Criança. A população foi constituída por crianças de 1 a 6 meses utilizando métodos audiovisuais. Resultados: A filmagem se procedeu por idade em ordem crescente e com a presença dos pais, por conseguinte era feito a estimulação de cada marco para identificar se o mesmo estava presente, ausente ou não verificado. Conclusão: $O$ vídeo educativo constitui-se importante tecnologia na assistência de enfermagem e ferramenta do processo ensino aprendizagem, assim como contribui para melhoria da qualidade do cuidado com ênfase na valorização dos saberes.

Palavras-chave: Atenção Primária à Saúde; Desenvolvimento Infantil; Enfermagem.

\begin{abstract}
Objective: To report the experience of producing educational video describing the milestones of child development from 1 to 6 months, in order to promote training for nursing professionals in primary health care, aiming at the effective assessment of the milestones of child development. Methodology: Study of an experience report type developed in the Child Health discipline. The population consisted of children aged 1 to 6 months using audiovisual methods. Results: The filming was done by age in ascending order and with the presence of the parents, therefore the stimulation of each landmark was made to identify whether it was present, absent or not verified. Conclusion: The educational video is an important technology in nursing care and a tool in the teaching-learning process, as well as contributing to improving the quality of care with an emphasis on valuing knowledge.
\end{abstract}

Key Words: Primary Health Care; Child Development; Nursing.

\footnotetext{
${ }^{1}$ Estudante do Curso de Enfermagem. UFMA. E-mail: alinemm96@gmail.com

2 Estudante do Curso de Enfermagem. UFMA. E-mail: jessicamaysaolib@gmail.com

${ }^{3}$ Estudante do Curso de Enfermagem. UFMA. E-mail: lcnalvees35@gmail.com

${ }^{4}$ Orientadora. Mestre em Ciências da Saúde. Docente Florence. E-mail: sannamartins1021@gmail.com

${ }^{5}$ Estudante do Curso de Enfermagem. UFMA. E-mail: taycostascj@gmail.com
} 


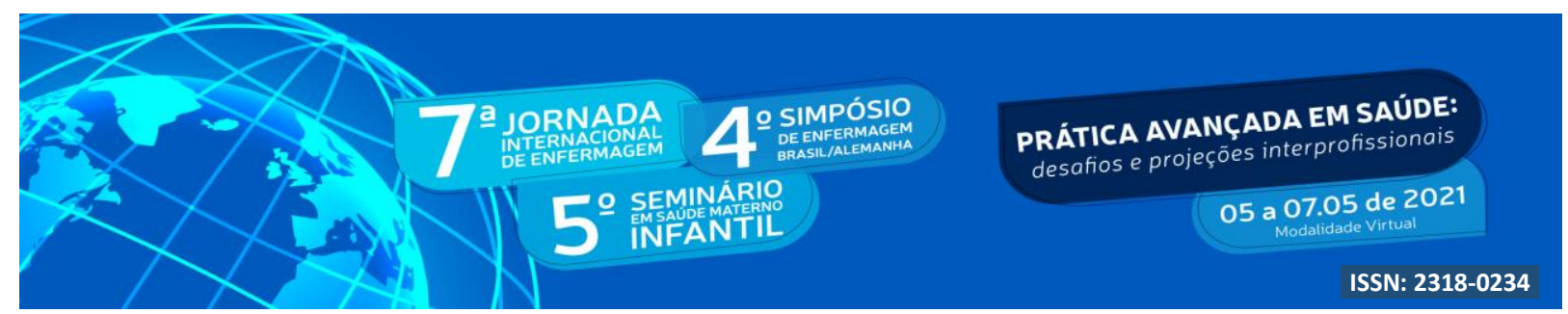

\section{INTRODUÇÃO}

O desenvolvimento infantil é a etapa mais importante da construção do ser humano, bem como é um dos eixos que define e qualifica o processo ativo e contínuo de vigilância à saúde das crianças, expresso por continuidade e mudanças nas habilidades motoras, cognitivas, psicossociais e de linguagem, com aquisições progressivamente mais complexas nas funções da vida diária e no exercício do papel social (SOUZA et al, 2018).

A atenção à saúde da criança no Brasil vem apresentando transformações ao longo do tempo, tendo como reflexo os avanços das diretrizes das políticas sociais, bem como a implementação de programas e políticas públicas de saúde, as quais alcançaram a diminuição da mortalidade infantil. Desde a instituição da Política Nacional de Atenção Integral à Saúde da Criança (PNAISC), em 2015, a atenção às ações de vigilância e estímulo ao pleno crescimento e desenvolvimento (CD) da criança esteve presente, tamanha a importância deste eixo estratégico para a prevenção de agravos, promoção da saúde infantil e realização de intervenções em tempo oportuno, nos casos em que possíveis desvios sejam identificados (ARAÚJO, 2014).

Nesse sentido, a Caderneta de Saúde da Criança (CSC), proposto pelo Ministério da Saúde do Brasil, aparece como ferramenta fundamental a ser utilizada para este acompanhamento por possibilitar o registro de informações pertinentes a este monitoramento e além disso favorecer maior valorização e apropriação do instrumento pela família, adesão e corresponsabilização pelas ações de vigilância da saúde dos seus filhos (ALMEIDA et al, 2016).

O acompanhamento do desenvolvimento da criança na Atenção Básica objetiva sua promoção, proteção e a detecção precoce de alterações passíveis de modificação que possam repercutir em sua vida futura. Isso ocorre principalmente por meio da vigilância do desenvolvimento e do acompanhamento integral. Entretanto, poucos profissionais de saúde, em especial os da Atenção Básica, praticam a vigilância do desenvolvimento e em muitos casos verifica-se que o conhecimento sobre o assunto é falho, bem como apresentam limitações tanto para entender o que é desenvolvimento, quanto para avaliá-lo a partir dos marcos definidos pelo Ministério da Saúde (ALVES et al, 2009; CIAMPO et al, 2006; 


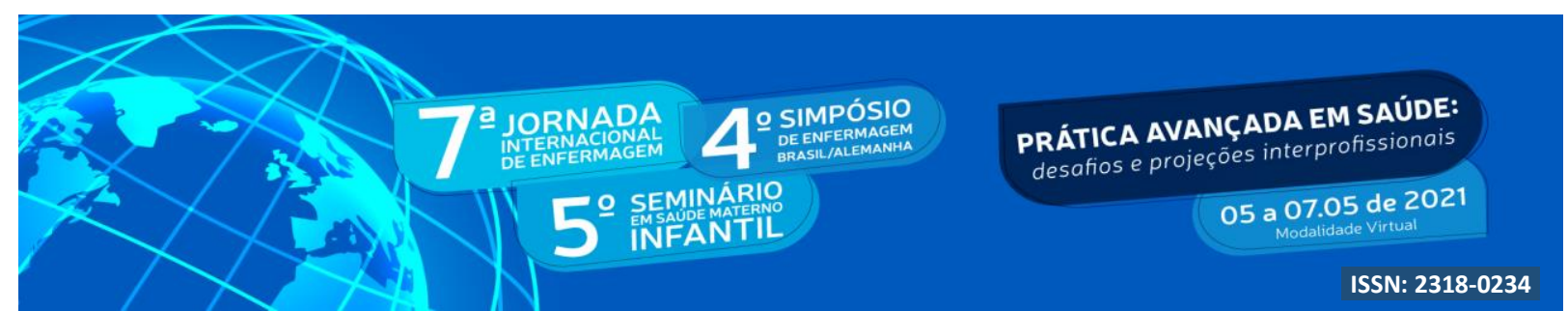

FIGUEIRAS et al, 2003).

Oliveira (2012) destaca que é preciso haver um melhor preparo dos profissionais que trabalham na atenção básica à saúde no Brasil em relação ao tema desenvolvimento infantil. A partir deste pressuposto questionou-se: Que recurso poderia ser disponibilizado para apoiar as práticas da formação profissional, visando a efetiva avaliação dos marcos do desenvolvimento infantil?

\section{OBJETIVO}

Relatar a experiência da produção de vídeo educativo descrevendo os marcos do desenvolvimento infantil de 1 a 6 meses, a fim de promover formação aos profissionais de enfermagem da atenção primária à saúde, visando a efetiva avaliação dos marcos do desenvolvimento infantil.

\section{METODOLOGIA}

Estudo do tipo relato de experiência desenvolvido na disciplina de Saúde da Criança entre os meses de setembro e novembro de 2019. A população foi constituída por crianças de 1 a 6 meses com autorização dos responsáveis para ser apresentado em sala de aula. O recurso tecnológico utilizado foi a elaboração de vídeo educativo direcionado aos profissionais de enfermagem da atenção primária à saúde, com 3 minutos e 51 segundos, produzido com câmera profissional canon e para a edição do vídeo fora utilizado o programa final cut.

\section{RESULTADOS E DISCUSSÃO}

$\mathrm{Na}$ construção do vídeo fora separado as crianças para que fossem filmadas uma por vez, bem como foi preparado um ambiente calmo e silencioso para favorecer qualidade ao vídeo. A filmagem se procedeu por idade em ordem crescente e com a presença dos pais, por conseguinte era feito a estimulação de cada marco para identificar se o mesmo estava presente, ausente ou não verificado.

Posteriormente, na edição de cada situação foi acrescentado as legendas de forma explicativa, como a seguir: no primeiro mês, o ideal é que se observe a postura: barriga para cima, braços e pernas fletidos, cabeça lateralizada, se a criança observa um rosto, reage ao 


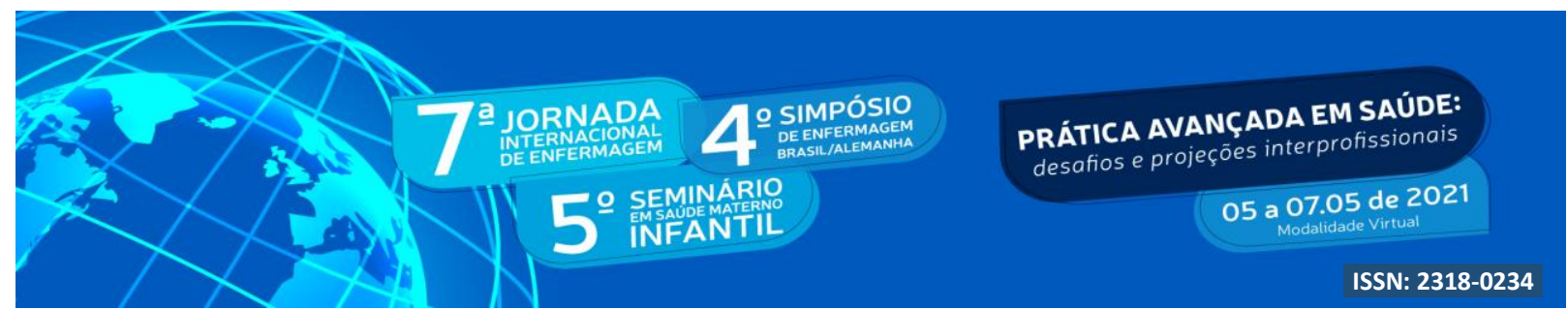

som, eleva a cabeça, apresenta sorriso social, abertura espontânea das mãos, emissão de sons e movimento ativo dos membros.

No segundo mês, além dos quatro últimos MD citados é esperado que a criança apresente resposta ativa ao contato social, que segure objetos e emita sons. No terceiro mês, como novidade, é previsto que de bruços, levante a cabeça e apoie-se nos antebraços e mantenha o contato social, pega de objetos e a emissão de sons.

No quarto mês ele deve manter todos os MD dos três meses e deve realizar a busca ativa de objetos, levar objetos à boca, localizar o som e mudar de posição ativamente (rolar). No quinto mês é desejável a busca ativa por objetos, levar objetos a boca, localizar o som e mudar de posição. Por fim, no sexto mês é esperado que se mantivesse os MD dos cinco meses e brinque de esconde-achou, duplique sílabas e sente-se sem apoio.

Logo após a essas descrições dos marcos foi intensificado a importância que o enfermeiro registre na caderneta e estabeleça a classificação da avaliação do desenvolvimento de acordo com a escala e os $\mathrm{MD}$ observados com $\mathrm{P}=$ marco presente, $\mathrm{A}=$ marco ausente $\mathrm{e}$ $\mathrm{NV}=$ marco não verificado, pois para classificação dos marcos é necessário que a criança se encaixe em alguma dessas categorias: provável atraso no desenvolvimento, alerta para o desenvolvimento, desenvolvimento adequado com fatores de risco e desenvolvimento adequado.

Nessa perspectiva, é atribuição do enfermeiro, por meio da vigilância do desenvolvimento infantil, prestar atenção qualificada, sistematizada, integral e individualizada. Nesse sentido, o cuidado à saúde da criança representa grande relevância na área de cuidados à saúde das populações, em decorrência da vulnerabilidade do ser humano nessa fase da vida (GAÍVA et al, 2018; VIEIRA et al, 2019).

$\mathrm{O}$ cuidado da criança implica em atender às necessidades essenciais para o seu desenvolvimento e o enfermeiro pode oferecer esses cuidados por meio da consulta de enfermagem com o objetivo de prevenir a ocorrência de agravos que podem prejudicar o pleno desenvolvimento infantil (VIEIRA et al, 2019).

A atuação do enfermeiro aborda conhecimentos científicos e a prática da sistematização de enfermagem para um bom acompanhamento do CD infantil. Dessa forma, o profissional deve conhecer os aspectos mais relevantes do desenvolvimento e estar preparado para intervir, se necessário, bem como identificar as crianças que precisam ser referidas para 


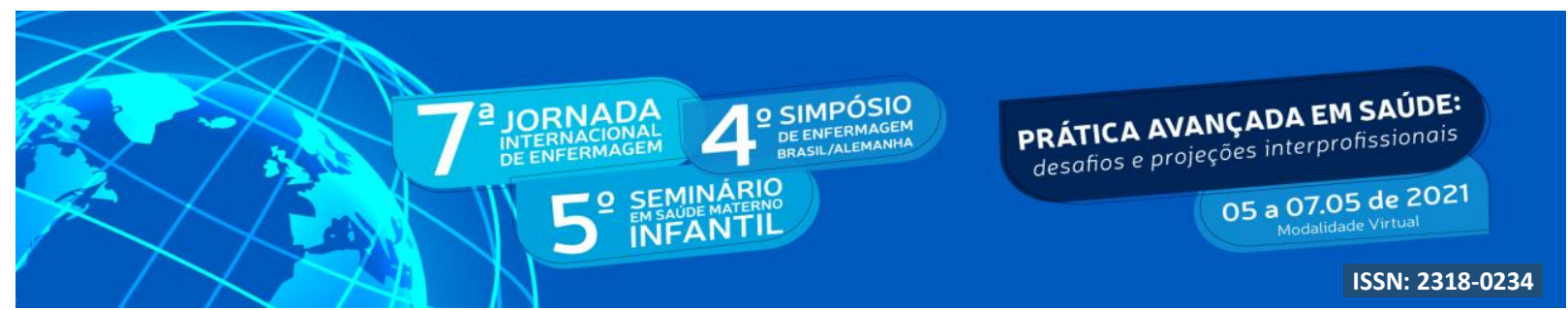

atendimento. Para uma boa avaliação do desenvolvimento infantil, é necessário que haja uma boa comunicação, criando uma relação de confiança entre a criança, mãe, família e comunidade, oferecendo um acolhimento adequado para a criança e familiares, proporcionar uma consulta de enfermagem com profissional qualificado, com atendimento adequado às necessidades individuais, com diálogos e orientações quanto aos cuidados da criança (GAÍVA et al, 2018).

\section{CONCLUSÃO}

Boa parte dos profissionais de saúde, segundo pesquisas, demonstra pouco conhecimento na execução da avaliação dos marcos do desenvolvimento infantil. Tocante a isso, o enfermeiro é o profissional de referência e sua contribuição tem sido de alta relevância.

Um dos grandes desafios do enfermeiro dentro da assistência do CD é estabelecer um vínculo de confiança entre a criança, mãe e familiares, a fim de garantir assiduidade nas consultas de enfermagem, e assegurar que a criança cresça e se desenvolva de forma saudável e segura dentro da sociedade. Entretanto, a assistência de enfermagem é importante no estabelecimento do cuidado respeitoso, acolhedor e que forneça algum vínculo de confiança entre os usuários.

Por fim, o vídeo educativo constitui-se importante tecnologia na assistência de enfermagem e ferramenta do processo ensino aprendizagem, assim como contribui para melhoria da qualidade do cuidado com ênfase na valorização dos saberes. Espera-se, a partir da exibição do vídeo, que os profissionais sejam capazes de executar corretamente a avaliação dos marcos do desenvolvimento infantil e preenchimento dos mesmos na CSC. Além disso, aponta-se a necessidade desses profissionais compreenderem as consequências de um diagnóstico e tratamento tardio para a qualidade de vida das crianças, na tentativa de sensibilizá-los para a efetivação da vigilância do desenvolvimento na atenção primária à saúde.

\section{REFERÊNCIAS}

Almeida, A. C. et al. Uso de instrumento de acompanhamento do crescimento e desenvolvimento da criança no Brasil - Revisão sistemática de literatura. Revista Paulista de

Pediatria. $\quad$ v. $34, \quad$ n. $1, \quad$ p. 122-31, 2016. Disponível em:< 


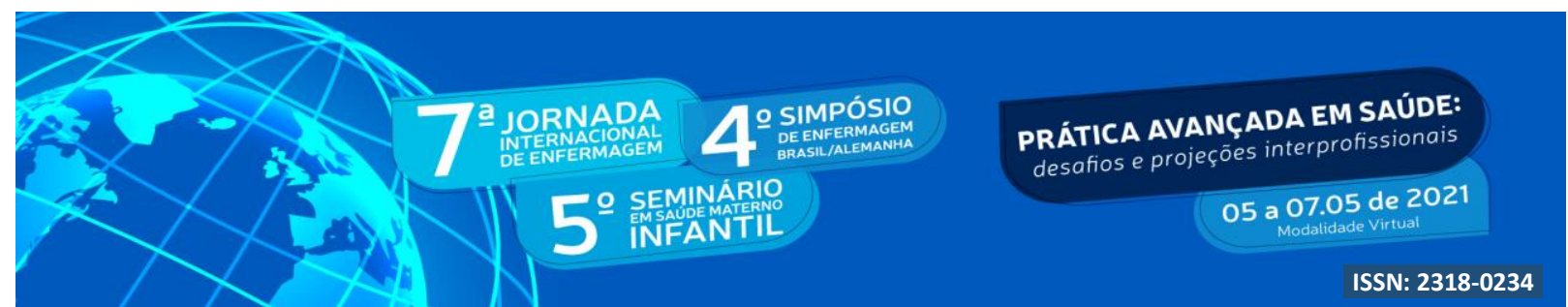

https://www.scielo.br/pdf/rpp/v34n1/pt_0103-0582-rpp-34-01-0122.pdf>. Acesso em 12 de marc. 2021.

ALVES, C. R. et al. Qualidade do preenchimento da Caderneta de Saúde da Criança e fatores associados. Cadernos de Saúde Pública, Rio de Janeiro, v.25, n.3, p. 583-595, 2009. Disponível em:< https://www.scielosp.org/pdf/csp/2009.v25n3/583-595/pt. Acesso em 13 de marc. 2021.

ARAÚJO, J. P. et al. História da saúde da criança: conquistas, políticas e perspectivas. Rev Bras Enferm. v. 67, n. 6: 1000-7, nov-dez, 2014. Disponível em:< https://www.scielo.br/pdf/reben/v67n6/0034-7167-reben-67-06-1000.pdf >. Acesso em 12 de marc. 2021.

BRASIL, Gabinete do Ministro. Portaria ${ }^{\circ}$ 1.130, de 5 de agosto de 2015. Institui a Política Nacional de Atenção Integral à Saúde da Criança (PNAISC) no âmbito do Sistema Único de Saúde (SUS) [Internet]. Brasília: Ministério da Saúde; 2015 [citado em 1 de fevereiro de 2019]. Disponível em: $<$ http://bvsms.saude.gov.br/bvs/saudelegis/gm/2015/prt1130_05_08_2015.html>. Acesso em 12 de marc. 2021.

CIAMPO, L. A. D. Et al. O Programa de Saúde da Família e a Puericultura. Ciênc Saúde Coletiva. v. 11, n. 3, p. 739-43, 2006. Disponível em:< https://www.scielo.br/pdf/csc/v11n3/30988.pdf>. Acesso em 13 de marc. 2021.

FIGUEIRAS, A. C. M. et al. Avaliação das práticas e conhecimentos de profissionais da atenção primária à saúde sobre vigilância do desenvolvimento infantil. Cadernos de Saúde Pública, Rio de Janeiro, v. 19, n.6, p.1691-1699, 2003. Disponível em:< https://www.scielo.br/pdf/csp/v19n6/a13v19n6.pdf>. Acesso em 13 de marc. 2021.

GAÍVA, M. A. M., MONTESCHIO, C. A. C, MOREIRA, M. D. S, SAlGE, A. K. M. Avaliação do crescimento e desenvolvimento infantil na consulta de enfermagem. Av Enferm., v. 36, n. 1, p. 9-21, 2018. Disponível em:< http://www.scielo.org.co/pdf/aven/v36n1/0121-4500-aven-36-01-00009.pdf. Acesso em 22 de marc. 2021.

OLIVEIRA, L. L. et al. Desenvolvimento infantil: concordância entre a caderneta de saúde da criança e o manual para vigilância do desenvolvimento infantil. Revista Paulista de Pediatria, São Paulo, v.30, n.4, p. 479-485, 2012. Disponível em:< https://www.scielo.br/pdf/rpp/v30n4/04.pdf>. Acesso em 22 de marc. 2021.

SOUZA, M. A. F. et al. Construção e validação de tecnologia comportamental para acompanhamento dos marcos do desenvolvimento infantil. Rev Rene. 2018;19:e33808. Disponível em:< http://periodicos.ufc.br/rene/article/view/33808/pdf_1>. Acesso em 13 de marc. 2021. 


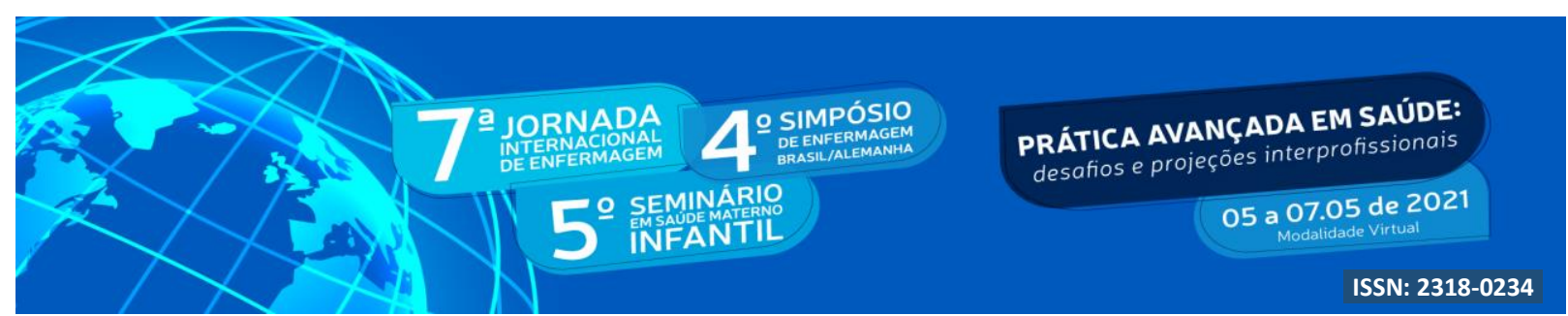

VIEIRA, Daniele de Souza et al. Processo de trabalho de enfermeiros na vigilância do desenvolvimento infantil. Rev Min Enferm., v. 23, 2019. Disponível em:< https://cdn.publisher.gn1.link/reme.org.br/pdf/e1242.pdf>. Acesso em 22 de marc. 2021. 